\title{
Extended Gari-Krümpelmann model fits to nucleon electromagnetic form factors
}

\author{
Earle L. Lomon \\ Center for Theoretical Physics \\ Laboratory for Nuclear Science and Department of Physics \\ Massachusetts Institute of Technology, Cambridge, Massachusetts 02139 \\ MIT-CTP-3111
}

Nucleon electromagnetic form factor data (including recent data) is fitted with models that respect the confinement and asymptotic freedom properties of QCD. Gari-Krümpelmann (GK) type models, which include the major vector meson pole contributions and at high momentum transfer conform to the predictions of perturbative QCD, are combined with Höhler-Pietarinen (HP) models, which also include the width of the $\rho$ meson and the addition of higher mass vector meson exchanges, but do not evolve into the explicit form of PQCD at high momentum transfer. Different parameterizations of the GK model's hadronic form factors, the effect of including the width of the $\rho$ meson and the addition of the next (in mass) isospin 1 vector meson are considered. The quality of fit and the consistency of the parameters select three of the combined HP/GK type models. Projections are made to the higher momentum transfers which are relevant to electron-deuteron experiments. The projections vary little for the preferred models, removing much of the ambiguity in electron-nucleus scattering predictions.

13.40.Gp, 21.10.Ft

\section{INTRODUCTION}

The electromagnetic form factors (emff) of the neutron and proton contain all the information about the charge and current distribution of these baryons, providing strong constraints on the fundamental theory of strong interactions. In addition the predictions for the emff of many-nucleon systems are sensitive to the input nucleon emff, as well as the many-body effects one would like to determine. For the first aspect one would like to have an accurate description of the data in a form closely linked to the fundamental theory. For the second it is convenient to have a simple analytic form to embed in the many-body calculation. In the past models with different physical constraints, equally well fitted to the available data, have predicted nucleon emff which differ sufficiently to induce large ambiguities in deuteron and heavier nucleus emff predictions. This has limited what can be learned about nuclear forces and meson-exhange current effects. The analysis here shows that, by combining the important physical features of past models and the data set now available, the few models which fit the data well, and with parameters most consistent with other reactions, produce small variations in the nucleon predicted emff over an extended range.

Accepting QCD as the fundamental theory of strong interactions, the emff can be described by perturbative QCD (PQCD) at very high momentum transfers. At low momentum 
transfers the confinement property of QCD implies an effective hadronic description with vector meson dominance (VMD, the coupling of the photon to vector mesons that couple in turn to the nucleons). Early models of the nucleon emff were based on VMD alone [1,2] including the $\rho, \omega$, and $\phi$ poles and the cut associated with the $\rho$ width, but with several phenomenological higher mass poles added. Gari and Krümpelmann [3] restricted the VMD contribution to the $\rho, \omega$, and $\phi$ poles, but added factors and terms which explicitly constrained the asymptotic momentum transfer behavior to the scaling behavior of PQCD. The additional factors, specified in the next section, are, in effect, hadronic form factors. We fit a series of four GK type models (varying only in the details of the hadronic form factors, as motivated in Section (II) to the present data set. In addition to the GK type models we consider a group of models (generically designated DR-GK) that use the analytic approximation of $[1$ to the dispersion integral approximation for the $\rho$ meson contribution (similar to that of HP [2]), modified by the hadronic form factors of the type we use with the GK model, and the addition of the $\rho^{\prime}$ (1450) pole. These additions result in a better fit to the data than we obtain with only the GK model [3] and minor variants of the hadronic form factors.

In this paper we fit the world data set for $G_{E p}, G_{M p}, G_{E n}, G_{M n}$ and $R_{p}=\mu_{p} G_{E p} / G_{M p}$. The last quantity, $R_{p}$, is a direct result of a recent measurement [5] with a polarized electron beam. We find similar results with the GK type models for three different parameterizations of the hadronic form factors (the fourth fits poorly), all three of the fits being reasonable when the inconsistency of the of the data, particularly for the neutron, is taken into account (some of the data sets must have large systematic errors, unless the emff oscillate over unnaturally small momentum transfer scales). With the extended DR-GK type models described above, qualitatively better fits are obtained for all four parameterizations of the hadronic form factors.

In Section 【I we will specify the models and parameters. Section [II will summarize the data set and the optimization procedure, while Section $\mathbb{\nabla}$ will present the results in comparison with each other and the original GK fit. We extrapolate beyond the present experimental range of momentum transfer where necessary for predicting available deuteron emff, and comment on the differences between the models in the extended range. For the three models with the lowest (nearly equal) $\chi^{2}$ fits to the data and parameters most consistent with other reactions, the differences are small. These DR-GK models are consistent with the requirements of dispersion relations and of QCD at low and high momentum transfer.

If these models are used as input in many-nucleon form factor calculations they will provide approximately stable results consistent with the nucleon data. Discrepancies with the many-nucleon data can then be attributed to deficiencies in the many-body wave function, meson exchange currents or relativistic corrections.

\section{NUCLEON EMFF MODELS}

The emff of a nucleon are defined by the matrix elements of the electromagnetic current $J_{\mu}$

$$
\left\langle N\left(p^{\prime}\right)\left|J_{\mu}\right| N(p)\right\rangle=e \bar{u}\left(p^{\prime}\right)\left\{\gamma_{\mu} F_{1}^{N}\left(Q^{2}\right)+\frac{i}{2 m_{N}} \sigma_{\mu \nu} Q^{\nu} F_{2}^{N}\left(Q^{2}\right)\right\} u(p)
$$


where $N$ is the neutron, $n$, or proton, $p$, and $-Q^{2}=\left(p^{\prime}-p\right)^{2}$ is the square of the invariant momentum transfer. $F_{1}^{N}\left(Q^{2}\right)$ and $F_{2}^{N}\left(Q^{2}\right)$ are respectively the Dirac and Pauli form factors, normalized at $Q^{2}=0$ as

$$
F_{1}^{p}(0)=1, \quad F_{1}^{n}(0)=0, \quad F_{2}^{p}(0)=\kappa_{p}, \quad F_{2}^{n}(0)=\kappa_{n} .
$$

Expressed in terms of the isoscalar and isovector electromagnetic currents

$$
2 F_{i}^{p}=F_{i}^{i s}+F_{i}^{i v}, \quad 2 F_{i}^{n}=F_{i}^{i s}-F_{i}^{i v}, \quad(i=1,2) .
$$

The Sachs form factors, most directly obtained from experiment, are then

$$
\begin{aligned}
& G_{\mathrm{EN}}\left(Q^{2}\right)=F_{1}^{N}\left(Q^{2}\right)-\tau F_{2}^{N}\left(Q^{2}\right) \\
& G_{\mathrm{MN}}\left(Q^{2}\right)=F_{1}^{N}\left(Q^{2}\right)+F_{2}^{N}\left(Q^{2}\right), \quad \tau=\frac{Q^{2}}{4 m_{N}} .
\end{aligned}
$$

The model of Gari and Krümpelmann [3] prescribes the following form for the four emff:

$$
\begin{aligned}
& F_{1}^{i v}\left(Q^{2}\right)=\frac{g_{\rho}}{f_{\rho}} \frac{m_{\rho}^{2}}{m_{\rho}^{2}+Q^{2}} F_{1}^{\rho}\left(Q^{2}\right)+\left(1-\frac{g_{\rho}}{f_{\rho}}\right) F_{1}^{D}\left(Q^{2}\right) \\
& F_{2}^{i v}\left(Q^{2}\right)=\kappa_{\rho} \frac{g_{\rho}}{f_{\rho}} \frac{m_{\rho}^{2}}{m_{\rho}^{2}+Q^{2}} F_{2}^{\rho}\left(Q^{2}\right)+\left(\kappa_{v}-\kappa_{\rho} \frac{g_{\rho}}{f_{\rho}}\right) F_{2}^{D}\left(Q^{2}\right) \\
& F_{1}^{i s}\left(Q^{2}\right)=\frac{g_{\omega}}{f_{\omega}} \frac{m_{\omega}^{2}}{m_{\omega}^{2}+Q^{2}} F_{1}^{\omega}\left(Q^{2}\right)+\frac{g_{\phi}}{f_{\phi}} \frac{m_{\phi}^{2}}{m_{\phi}^{2}+Q^{2}} F_{1}^{\phi}\left(Q^{2}\right)+\left(1-\frac{g_{\omega}}{f_{\omega}}\right) F_{1}^{D}\left(Q^{2}\right) \\
& F_{2}^{i s}\left(Q^{2}\right)=\kappa_{\omega} \frac{g_{\omega}}{f_{\omega}} \frac{m_{\omega}^{2}}{m_{\omega}^{2}+Q^{2}} F_{2}^{\omega}\left(Q^{2}\right)+\kappa_{\phi} \frac{g_{\phi}}{f_{\phi}} \frac{m_{\phi}^{2}}{m_{\phi}^{2}+Q^{2}} F_{2}^{\phi}\left(Q^{2}\right)+\left(\kappa_{s}-\kappa_{\omega} \frac{g_{\omega}}{f_{\omega}}-\kappa_{\phi} \frac{g_{\phi}}{f_{\phi}}\right) F_{2}^{D}\left(Q^{2}\right)
\end{aligned}
$$

where the pole terms are those of the $\rho, \omega$, and $\phi$ mesons, and the final term of each equation is determined by the asymptotic properties of PQCD. The $F_{i}^{\alpha}, \alpha=\rho, \omega$, or $\phi$ are the meson-nucleon form factors, while the $F_{i}^{D}$ are effectively quark-nucleon form factors.

In the final form used by GK, called Model 3 in [3], the above hadronic form factors are parameterized in the following way:

$$
\begin{aligned}
F_{1}^{\alpha, D}\left(Q^{2}\right) & =\frac{\Lambda_{1, D}^{2}}{\Lambda_{1, D}^{2}+\tilde{Q}^{2}} \frac{\Lambda_{2}^{2}}{\Lambda_{2}^{2}+\tilde{Q}^{2}} \\
F_{2}^{\alpha, D}\left(Q^{2}\right) & =\left(\frac{\Lambda_{1, D}^{2}}{\Lambda_{1, D}^{2}+\tilde{Q}^{2}}\right)^{2} \frac{\Lambda_{2}^{2}}{\Lambda_{2}^{2}+\tilde{Q}^{2}} \\
F_{1}^{\phi}\left(Q^{2}\right) & =F_{1}^{\alpha}\left(\frac{Q^{2}}{\Lambda_{1}^{2}+Q^{2}}\right)^{1.5}, \quad F_{1}^{\phi}(0)=0 \\
F_{2}^{\phi}\left(Q^{2}\right) & =F_{2}^{\alpha}\left(\frac{\Lambda_{1}^{2}}{\mu_{\phi}^{2}} \frac{Q^{2}+\mu_{\phi}^{2}}{\Lambda_{1}^{2}+Q^{2}}\right)^{1.5} \\
\text { with } \tilde{Q}^{2} & =Q^{2} \frac{\ln \left[\left(\Lambda_{2}^{2}+Q^{2}\right) / \Lambda_{\mathrm{QCD}}^{2}\right]}{\ln \left(\Lambda_{2}^{2} / \Lambda_{\mathrm{QCD}}^{2}\right)}, \quad \text { where } \alpha=\rho, \omega .
\end{aligned}
$$


This parameterization, together with Eq. (5), guarantees that the normalization conditions of Eq. (2) are met and that asymptotically

$$
\begin{aligned}
F_{1}^{i} & \sim\left[Q^{2} \ln \left(Q^{2} / \Lambda_{\mathrm{QCD}}^{2}\right)\right]^{-2} \\
F_{2}^{i} & \sim F_{1}^{i} / Q^{2} \\
i & =i s, i v
\end{aligned}
$$

as required by PQCD. When fitted to the data set described in section III, the result is here called Model GK(3).

In their Model 1 (fitted only to the proton data) GK associated the helicity flip hadronic form factors, $F_{2}$, with the quark-gluon scale cut-off $\Lambda_{2}$. However in model 3 , in fitting to the available data, they chose to associate the helicity flip with the meson scale cut-off $\Lambda_{1}$, as incorporated in Eq. (6). To investigate the effect of this change we also fit our data set, in Model GK(1), with the hadronic form factors of GK Model 1, for which

$$
F_{2}^{\alpha, D}=\frac{\Lambda_{1, D}^{2}}{\Lambda_{1, D}^{2}+\tilde{Q}^{2}}\left(\frac{\Lambda_{2}^{2}}{\Lambda_{2}^{2}+\tilde{Q}^{2}}\right)^{2}
$$

replaces the expressions in Eq. (6).

In both of the above parameterizations the logarithmic $Q^{2}$ dependence of PQCD is approached through a form factor determined by the $\Lambda_{2}$ and $\Lambda_{\mathrm{QCD}}$ cut-offs. In our Model GK'(1) we replace $\Lambda_{2}$ with $\Lambda_{D}$ for that factor which relates to the quark-nucleon vertex:

$$
\tilde{Q}^{2}=Q^{2} \frac{\ln \left[\left(\Lambda_{D}^{2}+Q^{2}\right) / \Lambda_{\mathrm{QCD}}^{2}\right]}{\ln \left(\Lambda_{D}^{2} / \Lambda_{\mathrm{QCD}}^{2}\right)} .
$$

Otherwise Model $\mathrm{GK}^{\prime}(1)$ is the same form as Model GK(1). A similar replacement was attempted for Model GK(3), but the best fit was substantially worse with the modification.

The next group of models replaces the $\rho$ meson pole terms in $F_{1}^{(i v)}$ and $F_{2}^{(i v)}$ (Eq. 5)with the well established $\rho^{\prime}$ (1450) meson pole term, and adds the $\rho$ meson term from the dispersion relation in approximate analytic form [1:

$$
\begin{aligned}
F_{1}^{i v}\left(Q^{2}\right)=N & \frac{1.0317+0.0875\left(1+Q^{2} / 0.3176\right)^{-2}}{\left(1+Q^{2} / 0.5496\right)} F_{1}^{\rho}\left(Q^{2}\right) \\
& +\frac{g_{\rho^{\prime}}}{f_{\rho^{\prime}}} \frac{m_{\rho^{\prime}}^{2}}{m_{\rho^{\prime}}^{2}+Q^{2}} F_{1}^{\rho}\left(Q^{2}\right)+\left(1-1.1192 N-\frac{g_{\rho^{\prime}}}{f_{\rho^{\prime}}}\right) F_{1}^{D}\left(Q^{2}\right) \\
F_{2}^{i v}\left(Q^{2}\right)=N & \frac{5.7824+0.3907\left(1+Q^{2} / 0.1422\right)^{-1}}{\left(1+Q^{2} / 0.5362\right)} F_{2}^{\rho}\left(Q^{2}\right) \\
& +\kappa_{\rho^{\prime}} \frac{g_{\rho^{\prime}}}{f_{\rho^{\prime}}} \frac{m_{\rho^{\prime}}^{2}}{m_{\rho^{\prime}}^{2}+Q^{2}} F_{2}^{\rho}\left(Q^{2}\right)+\left(\kappa_{\nu}-6.1731 N-\kappa_{\rho^{\prime}} \frac{g_{\rho^{\prime}}}{f_{\rho^{\prime}}}\right) F_{2}^{D}\left(Q^{2}\right)
\end{aligned}
$$

For $N=1$ the numerical values in Eq. 10 are those of [4] and are similar to those of [2]. They are determined by pion form factor and pion-nucleon p-wave phase shift input into 
the dispersion relation [4]. Because this input has uncertainties and is truncated at high momentum transfer, we considered the effect of an overall normalization factor $\mathrm{N}$ (the same for $F_{1}^{i v}$ and $F_{2}^{i v}$ ).

Because of the dispersion relation $\rho$ meson term, these models are labeled by DR-GK. Model DR-GK(3) has the hadronic form factors of Model GK(3) (Eq. (6)). Model DR-GK(1) uses the hadronic form factors of Model GK(1) (Eq. (8)). Model DR-GK'(1) and DR-GK'(3) are like Models DR-GK(1) and DR-GK(3) respectively but use the $\tilde{Q}^{2}$ of Eq. (9).

The best fit value of $N$ varied between 0.78 and 0.94 for these models, but $\chi^{2}$ decreased substantially only for model DR-GK(3). Consequently we present the results for the other three models with $N=1$, only introducing the extra parameter for Model DR-GK(3), now called DRN-GK(3).

\section{DATA BASE AND FITTING PROCEDURE}

The data for $G_{M_{p}}$ is from [6 [13]. The $G_{E_{p}}$ data is that of [6, [6,10,12 [14].

The data sources for $G_{M_{n}}$ are [12,15, 24]. The $G_{E_{n}}$ data is derived from 12, 18, 22, 23, [25 32]. Recent small revisions in the published values of [27,29,31] are included [33]. Quasielastic deuteron and ${ }^{3} \mathrm{He}$ data has been included, but the elastic deuteron data has been omitted because of its great sensitivity to the deuteron wave function. Another datum is the slope $d G_{E n} / d Q^{2}\left(Q^{2}=0\right)=0.0199 \pm 0.0003 \mathrm{fm}^{2}$, as determined by thermal neutron scattering [34].

The data set for the ratio $R_{p}$ includes not only [0], which measures the ratio directly in a polarization experiment, but also the data of [11], which extracts the ratio from unpolarized data dominated by the magnetic scattering.

There are 11 free parameters in each of the models; the three $g_{m} / f_{m}$ and the three $\kappa_{m}$ for the $\rho$ or $\rho^{\prime}, \omega$ and $\phi$ mesons, $\Lambda_{1}, \Lambda_{2}, \Lambda_{D}, \Lambda_{\mathrm{QCD}}$ and $\mu_{\phi}$. Model DRN-GK(3) has a 12th parameter, $N$. They were fitted by minimizing the value of $\chi^{2}$ for all the data using a Mathematica program that incorporates the Levenberg-Marquardt method.

\section{RESULTS}

Table II presents the "best fit" parameters to the present data set for the above 7 models. The parameters of [3] as fitted to the data set used in that reference are included in parentheses for Models GK(1) and GK(3). For all but two of the seven models "best fit" implies, as usual, the lowest local minimum in the search over the parameters. However for Models DRN-GK(3) and DR-GK'(3) the minimum is associated with indefinitely increasing negative values of $\kappa_{\rho}^{\prime}$. But $\chi^{2}$ decreases negligibly $(<1 \%)$ after reaching reasonable values of $\kappa_{\rho}^{\prime}$ which we choose to represent those two models.

As the models are simplifications of the actual physical situation, it is not required that the fitted parameters correspond to the values expected of them from measurement of independent observables. However those models for which the parameters are near those expectations are most consistent with the known physics. Only four of the models, GK(1), DRN-GK(3), DR-GK(1) and DR-GK'(1) have $\Lambda_{Q C D}$ in the range of 100-300 MeV consistent with high energy experiment. The value of $\kappa_{\rho}$ is only a free parameter for the three GK 
models. Its value is reasonable for GK(3) and $\mathrm{GK}^{\prime}(3)$ but is much too large for GK(1). Therefore only the above three DR-GK models are consistent with the expected values of both $\Lambda_{\mathrm{QCD}}$ and $\kappa_{\rho}$. Unfortunately none of the models have the expected small negative value of $\kappa_{\omega}$. This is probably indicative that at least one higher mass isoscalar meson is important to the form factor description (the $\omega$ and $\phi$ meson widths are too small to require a modification of the pole representation). Rather than further complicating the models, the isoscalar pole terms are to be regarded as effectively representing the more complicated situation of including higher mass isoscalar vector meson exchanges. The stability and adequacy of the fits is an indication that the form factors with more poles would be similar to those already obtained.

In Table \ the values of $\chi^{2}$ are listed for all the models and the contribution from each of the five form factor classes of measurement (see beginning of Section III) are detailed. For the GK type models $348.5<\chi^{2}<352.8$ and for the DR-GK type $322.5<\chi^{2}<327.1$. Therefore the quality of the fit is essentially the same within a model type, but the models that use the $\rho$ meson contribution as determined by dispersion relations (and substitute the parameterized $\rho^{\prime}$ pole contribution for the parameterized $\rho$ ) are significantly better fits to the data. Within a model type there are large differences in the fitted parameters and important differences in the distribution of $\chi^{2}$ contributions among the different form factors, in spite of the small variation of the total values of $\chi^{2}$. But the $\chi^{2}$ contributions differ little for the three models, DRN-GK(3), DR-GK(1) and DR-GK'(1), favored by their physical values of $\Lambda_{Q C D}$ and the dispersion representation of the $\rho$ meson contribution. We also note that while Model DRN-GK(3) has the smallest value of $\chi^{2}$, it is the only one incorporating a 12th parameter, the $\rho$ normalization $N$. With $N=1$ the best $\chi^{2}$ for this model is 375.1. By contrast the value of $\chi^{2}$ only decreases by 3 if $N$ is allowed to vary in the other three DR-GK type models.

We note that with the parameters of the original GK fit [3] the value of $\chi^{2}$ with respect to the present data set is 2.4 times larger than the best fit value for GK(1) and 3.0 times larger for GK(3). Therefore the data accumulated since 1992 has made an important difference. We also note that while the best fit values of $\chi^{2}$ are about twice the number of degrees of freedom, this excess is mostly due to clear inconsistencies in the data sets, most particularly for $G_{M n}$ at $Q^{2}<0.8 \mathrm{GeV} / c^{2}$. The displacement of nearby data points well beyond their given error bars is evident in the figures below. [4] quotes a $\chi^{2} /$ datum of 1.1. As their fit is similar to those given here, this disparity may be due not only to the data accumulated since 1995 but also to the compactification in their case of many low momentum transfer points into slopes of the form factors at the origin. Indeed, for the DRN-GK(3) model two points, at 0.33 and $0.81 \mathrm{GeV}^{2} / c^{2}$, deviate in opposite directions for $G_{E n}$ contributing 43.6 to a $\chi^{2}$ of 63.9. For the same model 8 points, ranging from 0.24 to $0.81 \mathrm{GeV}^{2} / c^{2}$, deviate from $G_{M n}$ with both signs and contribute 89.6 to a $\chi^{2}$ of 120.1 . The results are similar for the other models. This makes it clear that without the severe fluctuations of the experimental values outside their stated errors the fits presented here have achieved a value of $\chi^{2}$ close to the number of degrees of freedom.

The following Figs. 10 - 1 display the results for $G_{M p}, G_{E p}, R_{p}, G_{M n}$, and $G_{E n}$, in that order. $G_{E p}$ and $G_{E n}$ are normalized to the dipole form factor $G_{d}=\left(1+Q^{2} / 0.71\right)^{(-2)}$. $G_{M p}\left(G_{M n}\right)$ are normalized to the product of $G_{d}$ and $\mu_{p}\left(\mu_{n}\right)$. The Models GK(3), GK(1) and $\operatorname{GK}^{\prime}(1)$ as fitted to the present data are compared in Figs. 1(a)-5(a), while in Figs. 
1(b)-5(b) the same GK(3) and GK(1) are compared to those models with the parameters originally obtained in [3]. Figs. 1(c)-5(c) compare the results of Models DRN-GK(3), DRGK(1), DR-GK'(1) and DR-GK'(3) with the data. Figs. 6(a)-(c) show how all seven models extrapolate up to $Q^{2}=8 \mathrm{GeV}^{2} / c^{2}$ for $R_{p}$ and the neutron form factors, for which data is now restricted to $Q^{2}<4 \mathrm{GeV}^{2} / c^{2}$. For those observables we may expect data at higher momentum transfers in the near future.

Figs. 7(a)-(d) show $G_{M p}, G_{E p}, G_{M n}$, and $G_{E n}$ respectively for the three favored models in the reduced range $Q^{2}<2 \mathrm{GeV}^{2} / c^{2}$ where the data was very crowded in the previous figures. This gives a better view of the model differences and the scatter of experimental points at low $Q^{2}$.

For $G_{M p}$ (Fig. [1) all the models agree closely over the very large momentum transfer range up to $31 \mathrm{GeV} / c^{2}$. As shown in Fig. 1(b) even the substantial change in the fitted parameters from those of [3], which cause major differences in other form factors, make only a moderate difference here. But it should be noted that GK(3)-original is substantially lower at the peak near $2.5 \mathrm{GeV} / c^{2}$ than all the other models. In this same momentum transfer region there is also a dichotomy in the experimental points. There are some that peak near 1.06 ( [6, 11]) and others that peak near 1.03 ( [7,8]). The fits of all the present models favor the higher values. We also note (Fig. 7(a)) that the model DRN-GK(3) is slightly favored by the data for $Q^{2}<0.5 \mathrm{GeV}^{2} / c^{2}$.

The three GK type models are very close for $G_{E p}$, while the four DR-GK type models have more spread at $Q^{2}>5 \mathrm{GeV} / c^{2}$ (but still insignificant compared to experimental errors in that region). For $G_{E p}$, GK(3)-original is remarkable for its divergence from the present fits and GK(1)-original. This may be due to an emphasis in [3] on fitting the data of [7] at $Q^{2}$ of $2.003,2.497$, and $3.007 \mathrm{GeV}^{2} / c^{2}$, which were published shortly before [3] and in part motivated the variation of the GK(3) parameterization from that of GK(1). This data is substantially higher in value than other data sets in the same range of momentum transfer [6. 11.,I3] that were published earlier. It is to be noted that at very low $Q^{2}<0.3 \mathrm{GeV}^{2} / c^{2}$ the data [10] is systematically lower than the predictions of all the models (only the three favored models are in this expanded figure) and the trend of the data for $Q^{2}>0.3 \mathrm{GeV}^{2} / c^{2}$. If this old (1975) data is correct it implies that the models of charge distribution are inadequate at ranges beyond $0.5 \mathrm{fm}$.

The presented $R_{p}$ data in Figs. 3 is independent of the $G_{M p}$ and $G_{E p}$ data of Figs. 1 and 2. The experiment of [11] and the polarization data of [5], which measure this ratio directly, are included only in these figures. It is noticeable in comparing Figs. 3(a) and $3(\mathrm{c})$, and evident from the $\chi^{2}$ values (Table [II), that the DR-GK model fits are somewhat better than those of the GK models. Fig. 3(b) shows, as in the case of $G_{E p}$ discussed above, that the GK(3)-original model was too constrained by one particular set of data. The extrapolation of this fit to $8 \mathrm{GeV}^{2} / c^{2}$ for $R_{p}$, Fig. 6 (a), shows that this observable may be able to discriminate between the models at the higher $Q^{2}$ if the experimental errors do not increase at the higher momentum transfers. Even the models preferred for their fit and physical parameters, DRN-GK(3), DR-GK(1) and DR-GK'(1), differ by as much as 0.1 at $8 \mathrm{GeV}^{2} / c^{2}$.

Examining Figs. 4(a) and 4(c) one notes that while the overall fit to the $G_{M n}$ data is about the same for all models, the GK models converge near $Q^{2}=4 \mathrm{GeV}^{2} / c^{2}$ while the DR-GK models diverge there. Extrapolating to $8 \mathrm{GeV}^{2} / c^{2}$, Fig. $6(\mathrm{~b})$, the parameter 
favored models differ by almost 0.2 , an accuracy that may be more achievable experimentally than the above mentioned split for $R_{p}$. Fig. 7 (c) highlights the inconsistency of the $G_{M n}$ experiments for $Q^{2}<1.0 \mathrm{GeV}^{2} / c^{2}$

For $G_{E n}$ Figs. 5(a) and 5(c) show that the improved fit of the DR-GK over the GK type models is most evident at the higher $Q^{2}$. Extrapolating to $8 \mathrm{GeV}^{2} / c^{2}$, Fig. $6(\mathrm{c})$, there is little difference among the three parameter favored models. Again, the expanded Fig. 7(d) shows that there is considerable ambiguity in the low $Q^{2}$ data. In that figure we have added a new data point at $0.495 \mathrm{GeV}^{2} / \mathrm{CiteHZ}$ which became available too late to be included in the minimization of $\chi^{2}$. As the value is within 1 st. dev. of the model curves, its inclusion in the minimization would have made a negligible difference to the parameter fit.

\section{CONCLUSIONS}

Moderately good fits to the nucleon electromagnetic form factor data are achieved for seven variations and extensions of the Gari-Krümpelmann type model [3] which preserves VMD at low momentum transfers and PQCD behavior at high momentum transfers. The models all have simple analytic forms which are easily incorporated into few-nucleon formfactor predictions.

The four models which include the width of the $\rho$ meson, by use of dispersion relations, and the $\rho^{\prime}(1450)$ meson pole are a substantially better fit to the data than the $\rho, \omega$, and $\phi$ meson pole only GK models. The fitted parameters of three of the four, DRN-GK(3), DRGK(1), and DR-GK'(1), have values most compatible with independent evaluations. For these three models the predictions for the nucleon electromagnetic form factors are not only quantitatively similar over the range of the present experimental data, but differ little when $R_{p}, G_{M n}$, and $G_{E n}$ are extrapolated to $8 \mathrm{GeV}^{2} / c^{2}$. Consequently only small differences due to the nucleon form factors are expected in predictions of deuteron emff (for which there is already $A\left(Q^{2}\right)$ data up to $\left.Q^{2}=6 \mathrm{GeV}^{2} / c^{2}\right)$ and other few-nucleon electromagnetic form factors. This will eliminate a major ambiguity in the extraction of information about the few-nucleon wave functions and meson-exchange current effects. Precise data in the $Q^{2}=4-8 \mathrm{GeV}^{2} / c^{2}$ range may eventually further narrow the uncertainty.

\section{ACKNOWLEDGMENTS}

The author is grateful to Manfred Gari for discussion of the development of the model and to Haiyan Gao for information about new form factor data and re-evaluations of past data.

[1] F. Iachello, A.D. Jackson and A. Lande, Phys. Lett. B4, 191 (1973).

[2] G. Höhler et al., Nucl. Phys. B114, 505 (1976).

[3] M.F. Gari and W. Krümpelmann, Phys. Lett. B274, 159 (1992); erratum, Phys. Lett. B282, 483 (1992). 
[4] P. Mergell, Ulf-G. Meissner and D. Drechsel, Nucl. Phys. A596, 367 (1996).

[5] M.K. Jones et al., Phys. Rev. Lett. 84, 1398 (2000).

[6] L. Andivahis et al., Phys. Rev. D50, 5491 (1994).

[7] R.C. Walker et al., Phys. Rev. D49, 5671 (1994).

[8] A.F. Sill et al., Phys. Rev. D48, 29 (1993).

[9] P.E. Bosted et al., Phys. Rev. C42, 38 (1990).

[10] F. Borkowski et al., Nucl. Phys.B93, 461 (1975).

[11] W. Bartel et al., Nucl. Phys.B58, 429 (1973).

[12] K.M. Hanson et al., Phys. Rev.D8, 753 (1973).

[13] C.H. Berger et al., Phys. Lett.B35, 87 (1971).

[14] J.J. Murphy et al., Phys. Rev. C9, 2125 (1974).

[15] H. Anklin et al., Phys. Lett. B428, 248 (1998).

[16] E.E.W. Bruins et al., Phys. Lett. B36, 313 (1994).

[17] H. Gao et al., Phys. Rev. C50, R546 (1994).

[18] A. Lung et al., Phys. Rev. Lett. 70, 718 (1993).

[19] P. Markowitz et al., Phys. Rev. C48, R5 (1993).

[20] A.S. Esaulov et al. Sov. J. Nucl. Phys. 45, 258 (1987).

[21] H. Anklin et al., Phys. Lett. B336, 313 (1994).

[22] W. Bartel et al., Phys. Lett.B39, 407 (1972).

[23] W. Bartel et al., Phys. Lett.B30, 285 (1969).

[24] W. Xu et al., Phys. Rev. Lett. 85, 2900 (2000).

[25] J. Golack et al., Phys. Rev. C63, 034006 (2001).

[26] C. Herberg et al., Eur. Phys. J. A5, 131 (1999).

[27] M. Ostrick et al., Phys. Rev. Lett. 83, 276 (1999).

[28] I. Passchier et al., Phys. Rev. Lett. 82, 4988 (1999).

[29] D. Rohe et al., Phys. Rev. Lett. 83, 4257 (1999).

[30] T. Eden et al., Phys. Rev. C50, R1749 (1994).

[31] M. Meyerhoff et al., Phys. Lett.B327, 201 (1994).

[32] C.E. Jones-Woodward et al., Phys. Rev. C44, R571 (1999).

[33] T. Walcher, private communication.

[34] S. Kopecky et al., Phys. Rev. Lett. 74, 2927 (1995).

[35] H. Zhu et al., nucl-ex/0105001 


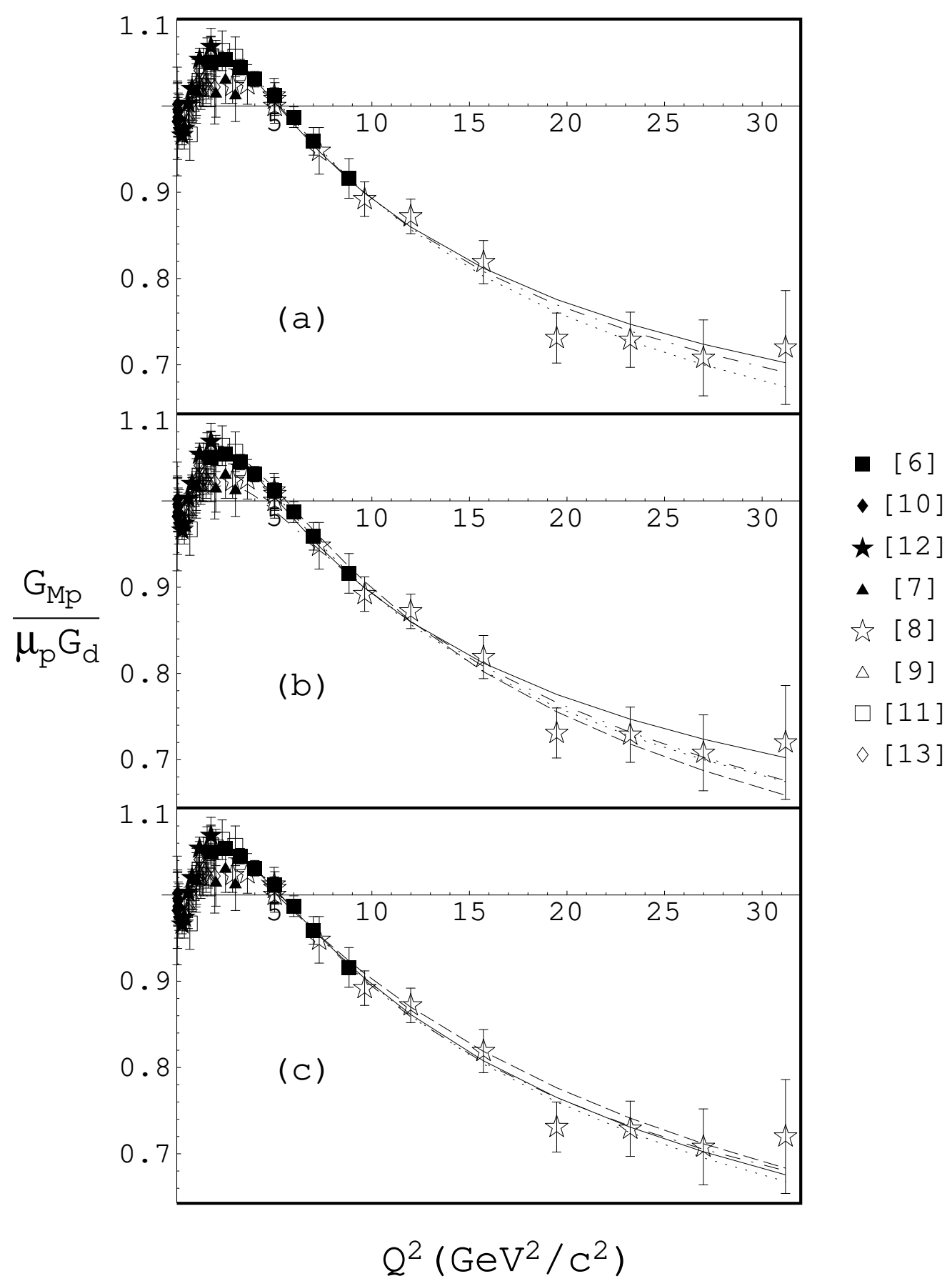

FIG. 1. $G_{M p}$ normalized to $\mu_{p} G_{d}$. (a) Comparison of the models GK(3) [solid], GK(1) [dotted] and $\mathrm{GK}^{\prime}(1)$ [dash-dotted] with the data. (b) Comparison of GK(3) [solid] and GK(1) [dotted] with the same models and the parameters of [3], GK(3)-original [dash-dotted] and GK(1)-original [dashed]. (c) Comparison of models DRN-GK(3) [solid], DR-GK(1) [dotted],

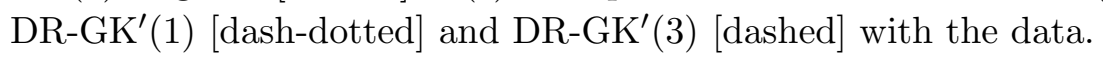




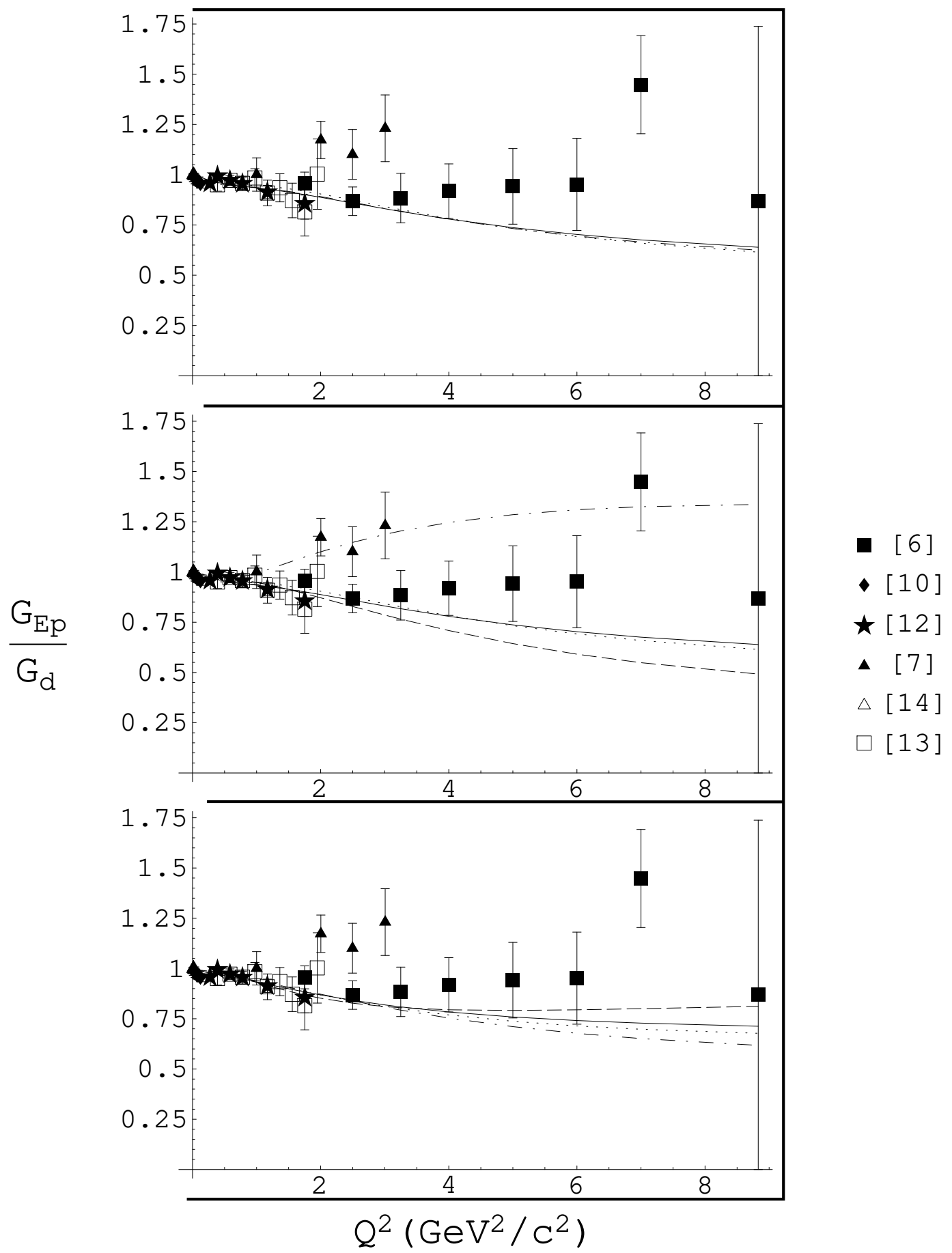

FIG. 2. $G_{E p}$ normalized to $G_{d}$. (a) Comparison of the models GK(3)[solid], GK(1)[dotted] and $\mathrm{GK}^{\prime}(1)$ [dash-dotted] with the data. (b) Comparison of GK(3)[solid] and GK(1)[dotted] with the same models and the parameters of [3], GK(3)-original[dash-dotted] and GK(1)-original[dashed]. (c) Comparison of models DRN-GK(3)[solid], DR-GK(1)[dotted], DR-GK'(1)[dash-dotted] and DR-GK'(3)[dashed] with the data. 


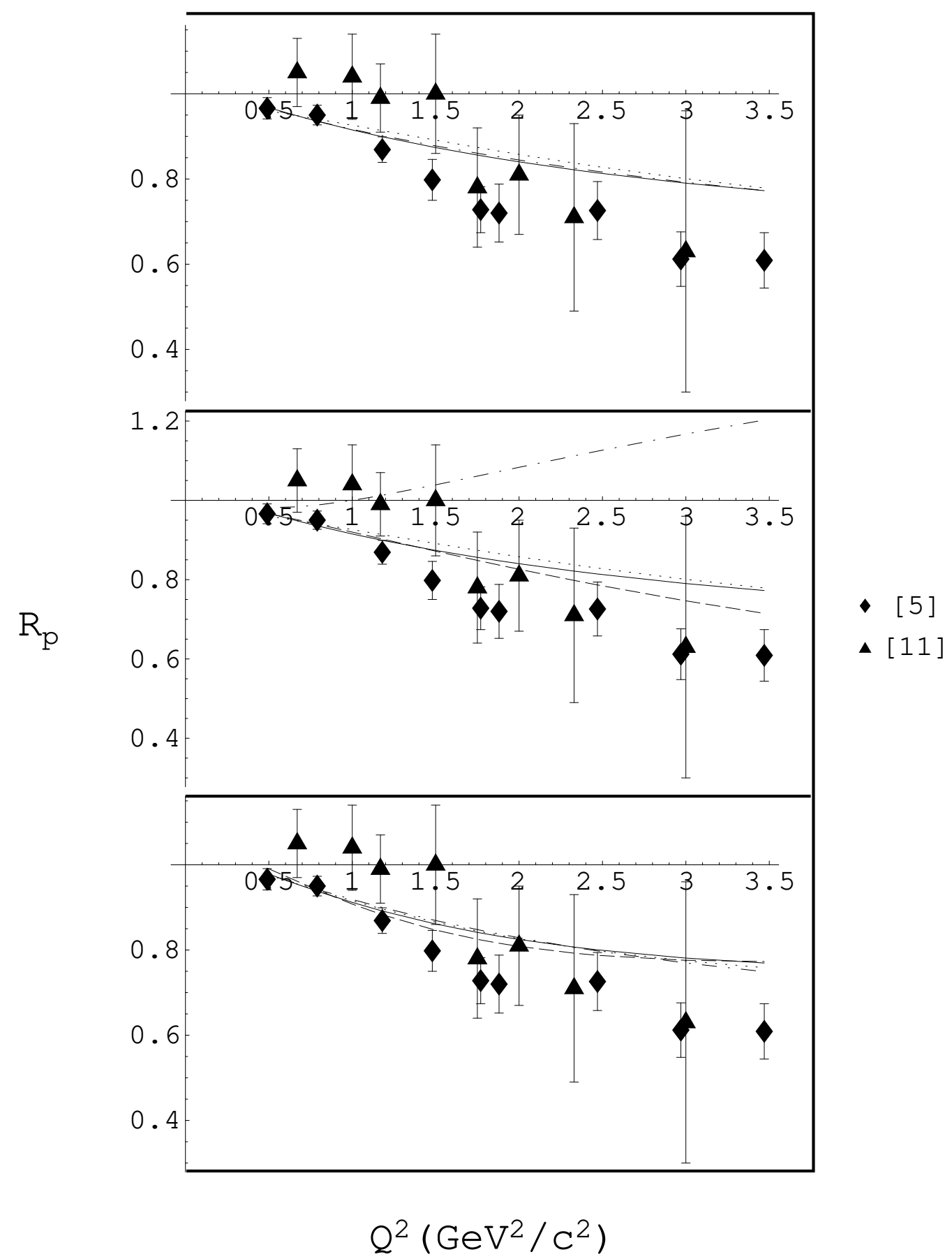

FIG. 3. $R_{p}$, the ratio $\mu_{p} G_{E p} / G_{M p}$. (a) Comparison of the models GK(3) [solid], GK(1) [dotted] and $\mathrm{GK}^{\prime}(1)$ [dash-dotted] with the data. (b) Comparison of GK(3) [solid] and GK(1) [dotted] with the same models and the parameters of [3], GK(3)-original [dash-dotted] and GK(1)-original [dashed]. (c) Comparison of models DRN-GK(3) [solid], DR-GK(1) [dotted], DR-GK'(1) [dashdotted] and DR-GK' $(3)$ [dashed] with the data. 


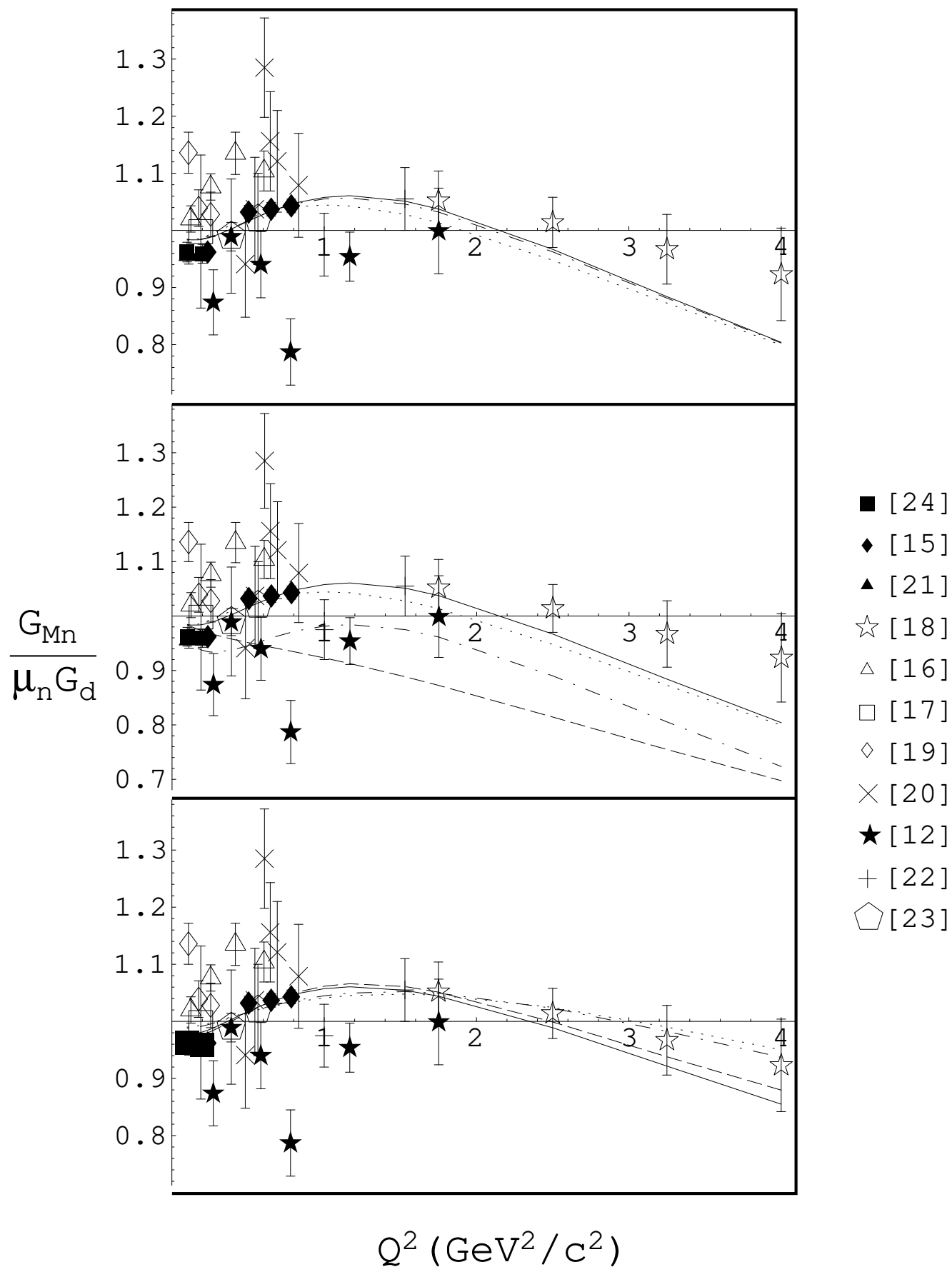

FIG. 4. $G_{M n}$ normalized to $\mu_{n} G_{d}$. (a) Comparison of the models GK(3) [solid], GK(1) [dotted] and $\mathrm{GK}^{\prime}(1)$ [dash-dotted] with the data. (b) Comparison of GK(3) [solid] and GK(1) [dotted] with the same models and the parameters of [3], GK(3)-original [dash-dotted] and GK(1)-original [dashed]. (c) Comparison of models DRN-GK(3) [solid], DR-GK(1) [dotted], $\mathrm{DR}^{-G_{K}^{\prime}}(1)$ [dash-dotted] and DR-GK'(3) [dashed] with the data. 


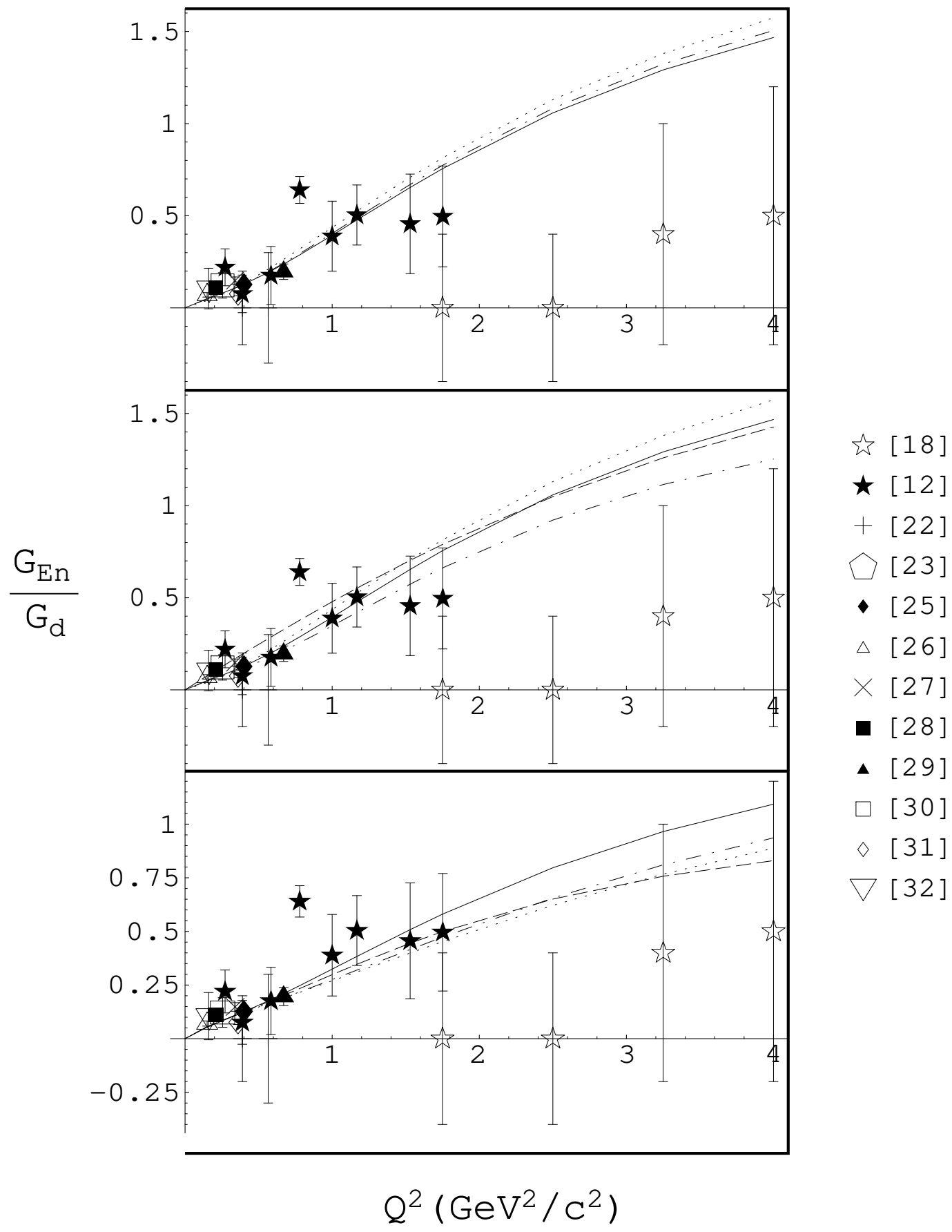

FIG. 5. $G_{E n}$ normalized to $G_{d}$. (a) Comparison of the models GK(3) [solid], GK(1) [dotted] and $\mathrm{GK}^{\prime}(1)$ [dash-dotted] with the data. (b) Comparison of GK(3) [solid] and GK(1) [dotted] with the same models and the parameters of [3], GK(3)-original [dash-dotted] and GK(1)-original [dashed]. (c) Comparison of models DRN-GK(3) [solid], DR-GK(1) [dotted], DR-GK'(1) [dash-dotted] and DR-GK' $(3)$ [dashed] with the data. 


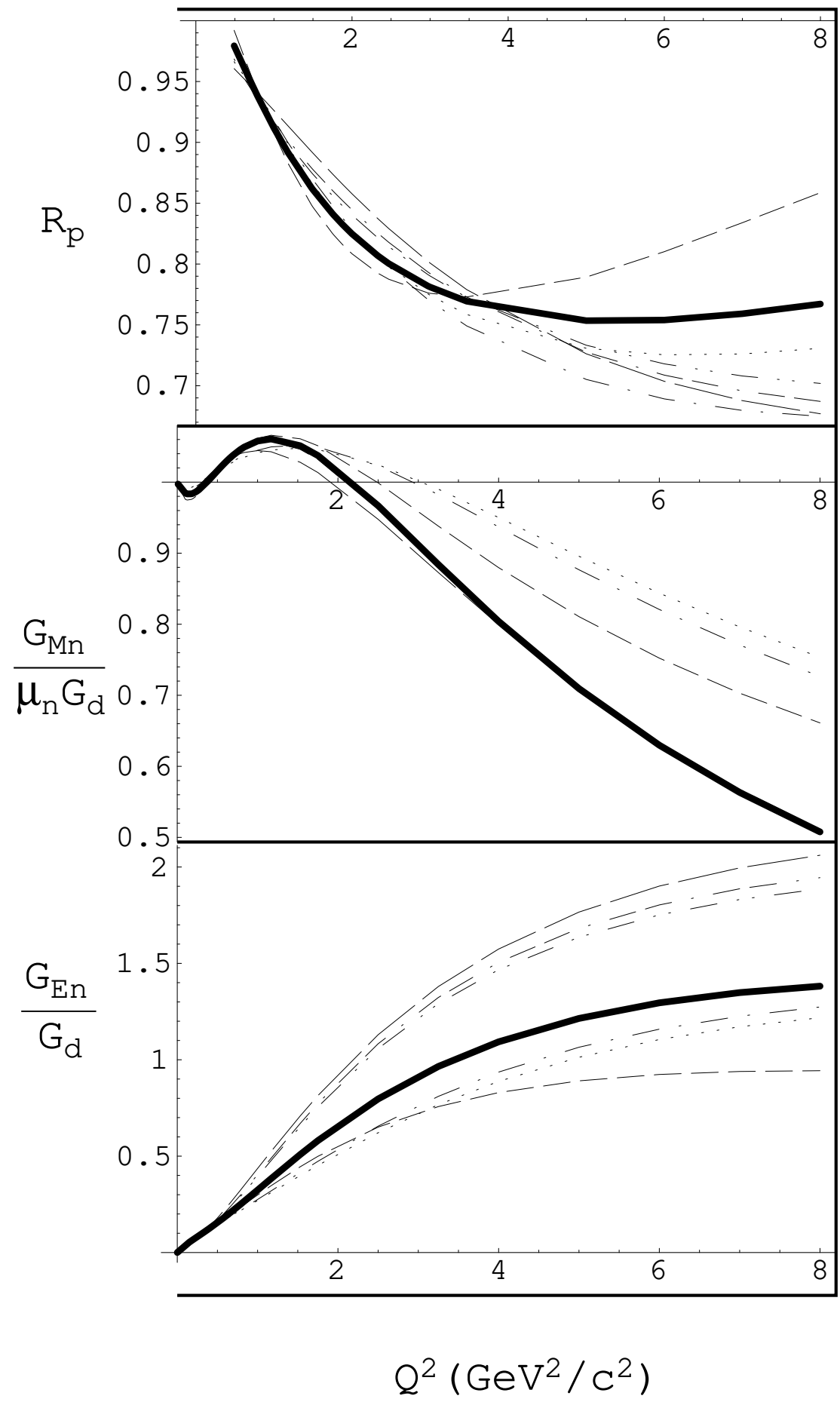

FIG. 6. Extrapolation to $Q^{2}=8 \mathrm{GeV}^{2} / c^{2}$. Comparison of the models DRN-GK(3) [solid], DR-GK(1) [dotted], DR-GK'(1) [dash-dotted], DR-GK'(3) [dashed], GK(3) [dash-double dotted], GK(1) [long dashes] and $\mathrm{GK}^{\prime}(1)$ [double dash-dotted]. (a) $R_{p}$, the ratio $\mu_{p} G_{E p} / G_{M p}$. (b) $G_{M n}$ normalized to $\mu_{n} G_{d}$. (c) $G_{E n}$ normalized to $G_{d}$. 


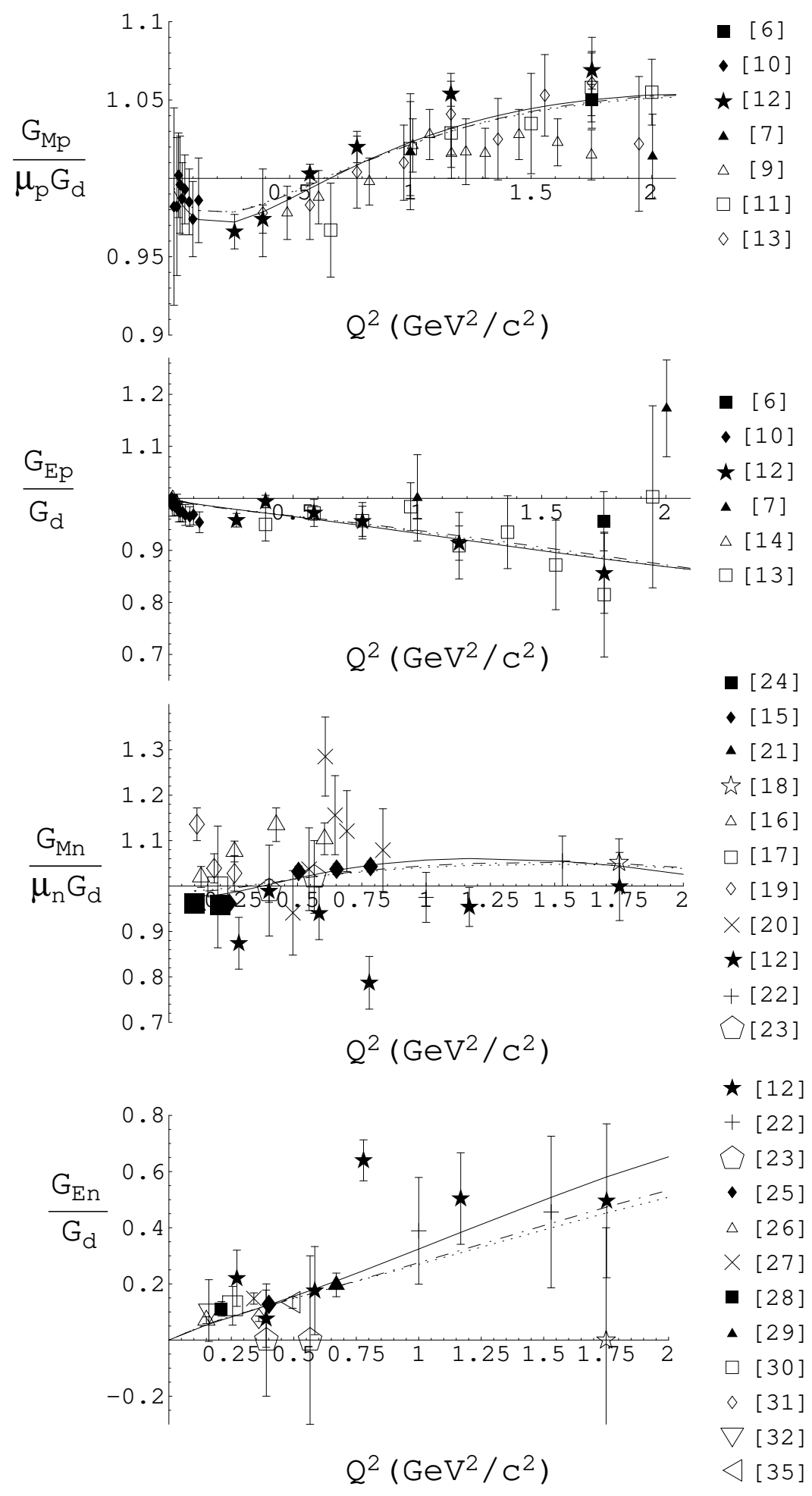

FIG. 7. Expanded interval $Q^{2}<2 \mathrm{GeV}^{2} / c^{2}$. Comparison of the favored models DRN-GK(3) [solid], DR-GK(1) [dotted], DR-GK'(1) [dash-dotted]. (a) $G_{M p}$ normalized to $\mu_{p} G_{d}$. (b) $G_{E p}$ normalized to $G_{d}$. (c) $G_{M n}$ normalized to $\mu_{n} G_{d}$. (d) $G_{E n}$ normalized to $G_{d}$. The point at 0.495 $\mathrm{GeV}^{2} / c^{2}$ [35] was added after optimization of the model parameters and appears only in this figure. 
TABLE I. Model parameters. Common to all models are $\kappa_{v}=3.706, \kappa_{s}=-0.12, m_{\rho}=0.776$ $\mathrm{GeV}, m_{\omega}=0.784 \mathrm{GeV}, m_{\phi}=1.019 \mathrm{GeV}$ and $m_{\rho^{\prime}}=1.45 \mathrm{GeV}$. Parentheses contain the values of [3].

\begin{tabular}{|c|c|c|c|c|c|c|c|}
\hline Parameters & & & & Models & & & \\
\hline & GK(3) & GK $(1)$ & $\mathrm{GK}^{\prime}(1)$ & DRN-GK(3) & DR-GK' $(3)$ & DR-GK(1) & DR-GK' $(1)$ \\
\hline (a) $g_{\rho^{(\prime)}} / f_{\rho^{(\prime)}}$ & $\begin{array}{c}0.4466 \\
(0.5688)\end{array}$ & $\begin{array}{l}0.0514 \\
(0.377)\end{array}$ & 0.3223 & 0.1013 & 0.0808 & 0.0625 & 0.0636 \\
\hline (a) $\kappa_{\rho^{(\prime)}}$ & $\begin{array}{l}4.3472 \\
(3.642)\end{array}$ & $\begin{array}{l}23.533 \\
(6.62)\end{array}$ & 4.982 & -15.870 & -17.993 & 0.9397 & -0.4175 \\
\hline$g_{\omega} / f_{\omega}$ & $\begin{array}{c}0.4713 \\
(0.5774)\end{array}$ & $\begin{array}{l}0.0588 \\
(0.411)\end{array}$ & 0.3440 & 0.6604 & 0.8038 & 0.8029 & 0.7918 \\
\hline$\kappa_{\omega}$ & $\begin{array}{c}21.762 \\
(0.4775)\end{array}$ & $\begin{array}{l}18.934 \\
(0.163)\end{array}$ & 40.661 & 8.847 & 4.0526 & 5.5225 & 5.1109 \\
\hline$g_{\phi} / f_{\phi}$ & $\begin{array}{l}-0.8461 \\
(-0.666)\end{array}$ & $\begin{array}{c}-0.5283 \\
(0.0)\end{array}$ & -0.9315 & -0.4054 & -0.2336 & -0.3070 & -0.3011 \\
\hline$\kappa_{\phi}$ & $\begin{array}{c}11.849 \\
(-0.2378)\end{array}$ & $\begin{array}{c}1.2236 \\
(0.0)\end{array}$ & 14.6805 & 13.6415 & 13.5963 & 14.4123 & 13.4385 \\
\hline$\mu_{\phi}$ & $\begin{array}{c}1.1498 \\
(0.33)\end{array}$ & $\begin{array}{c}1.1670 \\
(-)\end{array}$ & 1.1411 & 1.127 & 1.1218 & 1.2379 & 1.1915 \\
\hline$\Lambda_{1}$ & $\begin{array}{l}0.9006 \\
(0.823)\end{array}$ & $\begin{array}{l}0.5902 \\
(0.795)\end{array}$ & 0.8956 & 0.89361 & 0.9295 & 0.9916 & 0.9660 \\
\hline$\Lambda_{D}$ & $\begin{array}{l}1.7038 \\
(1.24)\end{array}$ & $\begin{array}{l}0.7273 \\
(0.795)\end{array}$ & 1.7038 & 1.0454 & 1.2207 & 1.2589 & 1.3406 \\
\hline$\Lambda_{2}$ & $\begin{array}{l}1.1336 \\
(1.95)\end{array}$ & $\begin{array}{l}1.9368 \\
(2.270)\end{array}$ & 0.9551 & 2.1614 & 3.9736 & 2.1327 & 2.1382 \\
\hline$\Lambda_{\mathrm{QCD}}$ & $\begin{array}{c}0.0312 \\
(0.31)\end{array}$ & $\begin{array}{l}0.1377 \\
(0.29)\end{array}$ & 0.0604 & 0.2452 & 0.4394 & 0.1377 & 0.1163 \\
\hline$N$ & - & - & - & 0.7838 & $1.0(\mathrm{~b})$ & $1.0(\mathrm{~b})$ & $1.0(\mathrm{~b})$ \\
\hline
\end{tabular}

(a) $\rho^{(\prime)}$ signifies the $\rho$ meson for the GK models and the $\rho^{\prime}(1450)$ meson for the DR-GK models. (b) not varied 
TABLE II. Contributions to the standard deviation, $\chi^{2}$, from each data type for each of the models. The number of data points, $n$, is listed for each data type. Parentheses contain results of ref. [3] parameters.

\begin{tabular}{|c|c|c|c|c|c|c|c|c|}
\hline $\begin{array}{r}\text { Data } \\
\text { type }\end{array}$ & $n$ & $\operatorname{GK}(3)$ & $\operatorname{GK}(1)$ & $\mathrm{GK}^{\prime}(1)$ & $\begin{array}{c}\text { Models } \\
\text { DRN-GK }(3)\end{array}$ & DR-GK'(3) & DR-GK(1) & $\operatorname{DR}^{-G^{\prime}}(1)$ \\
\hline$\overline{G_{M p}}$ & 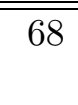 & $\begin{array}{c}47.6 \\
(206.8)\end{array}$ & $\begin{array}{c}45.4 \\
(71.9)\end{array}$ & 45.6 & $\begin{array}{ll}42.3\end{array}$ & 46.7 & $\begin{array}{ll}42.9 \\
\end{array}$ & 43 \\
\hline$G_{E p}$ & 48 & $\begin{array}{c}72.7 \\
(97.1)\end{array}$ & $\begin{array}{c}65.2 \\
(76.2)\end{array}$ & 71.8 & 65.8 & 68.0 & 65.8 & 67.2 \\
\hline$G_{M n}$ & 35 & $\begin{array}{c}124.8 \\
(344.1)\end{array}$ & $\begin{array}{c}123.9 \\
(393.9)\end{array}$ & 124.3 & 120.1 & 121.0 & 123.8 & 122.4 \\
\hline$G_{E n}$ & 23 & $\begin{array}{c}69.4 \\
(69.7)\end{array}$ & $\begin{array}{c}76.6 \\
(217.5)\end{array}$ & 70.5 & 63.9 & 62.8 & 65.1 & 64.8 \\
\hline$R_{p}$ & 17 & $\begin{array}{c}35.0 \\
(323.5)\end{array}$ & $\begin{array}{c}41.6 \\
(25.2)\end{array}$ & 36.4 & 30.5 & 27.8 & 29.4 & 29.0 \\
\hline Total & 191 & $\begin{array}{r}349.5 \\
(1,041.2)\end{array}$ & $\begin{array}{c}352.7 \\
(784.7)\end{array}$ & 348.6 & 322.6 & 326.3 & 327.0 & 326.7 \\
\hline
\end{tabular}

